\title{
QUENCHING PROBLEMS FOR A SEMILINEAR REACTION-DIFFUSION SYSTEM WITH SINGULAR BOUNDARY OUTFLUX
}

\author{
BURHAN SELCUK
}

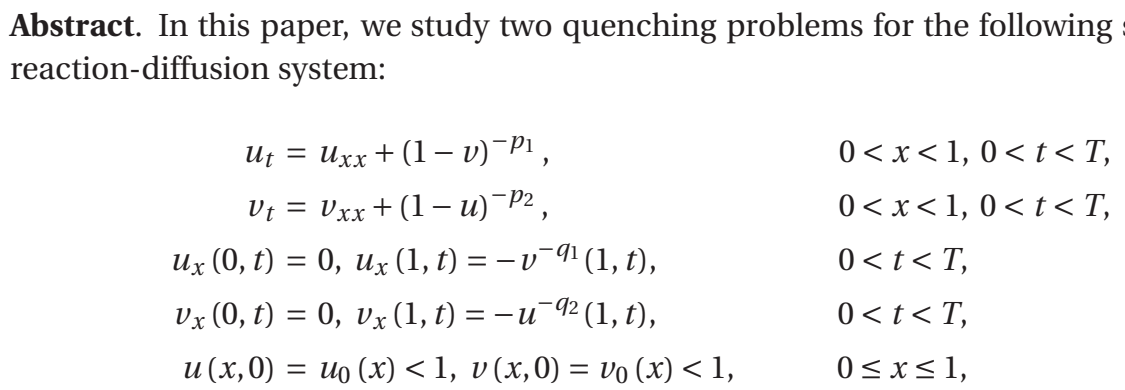

where $p_{1}, p_{2}, q_{1}, q_{2}$ are positive constants and $u_{0}(x), v_{0}(x)$ are positive smooth functions. We firstly get a local exisence result for this system. In the first problem, we show that quenching occurs in finite time, the only quenching point is $x=0$ and $\left(u_{t}, v_{t}\right)$ blows up at the quenching time under the certain conditions. In the second problem, we show that quenching occurs in finite time, the only quenching point is $x=1$ and $\left(u_{t}, v_{t}\right)$ blows up at the quenching time under the certain conditions.

\section{Introduction}

In this paper, we study two quenching problems for the following semilinear reactiondiffusion system:

$$
\begin{cases}u_{t}=u_{x x}+(1-v)^{-p_{1},} & 0<x<1,0<t<T, \\ v_{t}=v_{x x}+(1-u)^{-p_{2},} & 0<x<1,0<t<T, \\ u_{x}(0, t)=0, u_{x}(1, t)=-v^{-q_{1}}(1, t), & 0<t<T, \\ v_{x}(0, t)=0, v_{x}(1, t)=-u^{-q_{2}}(1, t), & 0<t<T, \\ u(x, 0)=u_{0}(x)<1, v(x, 0)=v_{0}(x)<1, & 0 \leq x \leq 1,\end{cases}
$$

Received June 24, 2015, accepted July 10, 2015.

2010 Mathematics Subject Classification. 35K51, 35K55, 35B50.

Key words and phrases. Reaction-diffusion system, singular boundary outflux, quenching, maximum principles, monotone iterations. 
where $p_{1}, p_{2}, q_{1}, q_{2}$ are positive constants and $u_{0}(x), v_{0}(x)$ are positive smooth functions satisfying the compatibility conditions

$$
u_{0}^{\prime}(0)=v_{0}^{\prime}(0)=0, u_{0}^{\prime}(1)=-v_{0}^{-q_{1}}(1), v_{0}^{\prime}(1)=-u_{0}^{-q_{2}}(1) .
$$

Our main purpose is to examine the quenching behavior of the solution of system (1.1) having different singular heat sources.

Definition 1. The solution of the problem (1.1) is said to quench if there exists a finite time $T$ such that

$$
\text { (a) } \lim _{t \rightarrow T^{-}} \max \{u(x, t), v(x, t): 0 \leq x \leq 1\} \rightarrow 1 \text {, }
$$

or

$$
\text { (b) } \lim _{t \rightarrow T^{-}} \min \{u(x, t), v(x, t): 0 \leq x \leq 1\} \rightarrow 0 .
$$

From now on, we denote the quenching time of the problem (1.1) with $T$.

The First Problem. The system (1.1) is supplemented with either the initial conditions

$$
\begin{gathered}
u_{x x}(x, 0)+(1-v(x, 0))^{-p_{1}} \geq 0(\neq 0), \\
v_{x x}(x, 0)+(1-u(x, 0))^{-p_{2}} \geq 0(\neq 0), \\
u_{x}(x, 0) \leq 0, v_{x}(x, 0) \leq 0 .
\end{gathered}
$$

Throughout this paper, we shall refer to the system (1.1), (1.2)-(1.3) conditions as the first problem.

The Second Problem. The system (1.1) is supplemented with either the initial conditions (1.3) and the inequalities

$$
\begin{aligned}
& u_{x x}(x, 0)+(1-v(x, 0))^{-p_{1}} \leq 0(\neq 0), \\
& v_{x x}(x, 0)+(1-u(x, 0))^{-p_{2}} \leq 0(\neq 0),
\end{aligned}
$$

Throughout this paper, we shall refer to the system (1.1), (1.3)-(1.4) conditions as the second problem.

Since 1975, quenching problems for the solutions of parabolic equations with various boundary conditions have been studied extensively ([1], [4], [8]). There are many papers considering the quenching phenomenon for the solutions of parabolic systems ([3], [9], [10]).

If $p_{1}=p_{2}, q_{1}=q_{2}$, and $u_{0}=v_{0}$, the problem (1.1) reduces to a following problem, namely

$$
\begin{cases}u_{t}=u_{x x}+(1-u)^{-p_{1}}, & (x, t) \in(0,1) \times(0, T), \\ u_{x}(0, t)=0, u_{x}(1, t)=-u^{-q_{1}}(1, t), & t \in(0, T), \\ u(x, 0)=u_{0}(x), & x \in[0,1] .\end{cases}
$$


Recently, Selcuk and Ozalp [8] studied the problem (1.5). They showed that $x=0$ is the quenching point in finite time, $\lim _{t \rightarrow T^{-}} u(0, t) \rightarrow 1$, if $u_{0}$ satisties $u_{x x}(x, 0)+(1-u(x, 0))^{-p} \geq 0$ and $u_{x}(x, 0) \leq 0$. Further they showed that $u_{t}$ blows up at quenching time. Furthermore, they obtained a quenching rate and a lower bound for the quenching time.

So far in literature, the quenching problem (a parabolic equation or a parabolic system) with different type two singular sources, which one is reaction term the other is absorption term, less studied. The quenching problem (1.1) with two type of singularity terms, namely, reaction terms $(1-v)^{-p_{1}},(1-u)^{-p_{2}}$ and the boundary outflux terms $-v^{-q_{1}},-u^{-q_{2}}$. Observe that in the problem (1.1) the singular source term may become singular if $u(x, t) \rightarrow$ $1^{-}, v(x, t) \rightarrow 1^{-}$as $(x, t) \rightarrow\left(x^{*}, T\right)$, where $x^{*}$ is a quenching point in $[0,1]$ and $T$ is a quenching time in $(0, \infty)$. On the other hand, the outflux $-v^{-q_{1}}(1, t),-u^{-q_{2}}(1, t)$ may also become singular in some finite time. Motivated by the problem (1.5) and [2], we discuss these situation in the present paper.

This paper is organized as follows. In Section 2, we obtain the local existence of the solution for problem (1.1). In Section 3, we consider the first problem. Firstly, we show that quenching, as definition 1 (a), occurs in finite time, only quenching point is $x=0$ and $\left(u_{t}, v_{t}\right)$ blows up at the quenching time under the conditions (1.2)-(1.3). In Section 4, we consider the second problem. We show that quenching, as definition 1 (b), occurs in finite time and the only quenching point is $x=1$ and $\left(u_{t}, v_{t}\right)$ blows up at the quenching time under the conditions (1.3)-(1.4).

\section{Local existence}

It is well known that one of the most effective methods to obtain existence and uniqueness results of the solutions of parabolic equations and systems with initial conditions is monotone iterative technique (for details see [1], [5] and [6]).

Let $C^{m}(Q), C^{\alpha}(Q)$ be the respective spaces of $m$-times differentiable and Hölder continuous functions in $Q$ with exponent $\alpha \in(0,1)$, where $Q$ is any domain. Denote by $C^{2,1}([0,1] \times$ $[0, T)$ ) the set of functions that are twice continuously differentiable in $x$ and once continuously differentiable in $t$ for $(x, t) \in[0,1] \times[0, T)$. It assumed that initial function $u_{0}(x)$ is in $C^{2+\alpha}$.

Definition 2. $(\widetilde{u}, \widetilde{v})$ is called an upper solution of the problem $(1.1)$ if $\widetilde{u}, \widetilde{v} \in C([0,1] \times[0, T)) \cap$ $C^{2,1}((0,1) \times(0, T))$ and $(\widetilde{u}, \widetilde{v})$ satisfies the following conditions:

$$
\begin{array}{rlrl}
\widetilde{u}_{t}-\widetilde{u}_{x x} \geq(1-\widetilde{v})^{-p_{1}}, & & 0<x<1,0<t<T, \\
\widetilde{v}_{t}-\widetilde{v}_{x x} \geq(1-\widetilde{u})^{-p_{2}}, & & 0<x<1,0<t<T, \\
\widetilde{u}_{x}(0, t) & =0, \widetilde{u}_{x}(1, t) \geq-\widetilde{v}^{-q_{1}}(1, t), & & 0<t<T,
\end{array}
$$




$$
\begin{array}{cc}
\widetilde{v}_{x}(0, t)=0, \widetilde{v}_{x}(1, t) \geq-\widetilde{u}^{-q_{2}}(1, t), & 0<t<T, \\
\widetilde{u}(x, 0) \geq u_{0}(x), \widetilde{v}(x, 0) \geq v_{0}(x), & 0 \leq x \leq 1 .
\end{array}
$$

Similarly, a lower solution of the problem (1.1), $\widehat{u}, \widehat{v} \in C([0,1] \times[0, T)) \cap C^{2,1}((0,1) \times(0, T))$, is defined by reversing the inequalities.

Lemma 1. Let $(\widetilde{u}, \widetilde{v})$ and $(\widehat{u}, \widehat{v})$ be a positive upper solution and a nonnegative lower solution of the problem (1.1) in $[0,1] \times[0, T)$, respectively. Then, we get following results;

(a) $\widetilde{u} \geq \widehat{u}$ and $\widetilde{v} \geq \widehat{v}$ in $[0,1] \times[0, T)$,

(b) if $\left(u^{*}, v^{*}\right)$ is a solution, then $\widetilde{u} \geq u^{*} \geq \widehat{u}$ and $\widetilde{v} \geq v^{*} \geq \widehat{v}$ in $[0,1] \times[0, T)$.

Proof. (a) We give the proof by utilizing Lemma 2.1 in [2]. Let $\Theta=\widetilde{u}-\widehat{u}$ and $\Psi=\widetilde{v}-\widehat{v}$. Then $\Theta(x, t)$ and $\Psi(x, t)$ satisfies

$$
\begin{array}{ll}
\Theta_{t} \geq \Theta_{x x}+a(x, t) \Psi, \Psi_{t} \geq \Psi_{x x}+b(x, t) \Theta, & 0<x<1,0<t<T, \\
\Theta_{x}(0, t) \leq 0, \Psi_{x}(0, t) \leq 0, & 0<t<T, \\
\Theta_{x}(1, t) \geq c(t) \Psi(1, t), \Psi_{x}(1, t) \geq d(t) \Theta(1, t), & 0<t<T, \\
\Theta(x, 0) \geq 0, \Psi(x, 0) \geq 0, & 0 \leq x \leq 1,
\end{array}
$$

where

$$
\begin{array}{rlrl}
a(x, t) & =\frac{(1-\widetilde{v}(x, t))^{-p_{1}}-(1-\widehat{v}(x, t))^{-p_{1}}}{\widetilde{v}(x, t)-\widehat{v}(x, t)}, & \text { if } \widetilde{v} \neq \widehat{v} ;=0, \text { otherwise, } \\
b(x, t)=\frac{(1-\widetilde{u}(x, t))^{-p_{2}}-(1-\widehat{u}(x, t))^{-p_{2}}}{\widetilde{u}(x, t)-\widehat{u}(x, t)}, & \text { if } \widetilde{u} \neq \widehat{u} ;=0 \text {, otherwise, } \\
c(t)=\frac{-\widetilde{v}^{-q_{1}}(1, t)-\left(-\widehat{v}^{-q_{1}}(1, t)\right)}{\widetilde{v}(1, t)-\widehat{v}(1, t)}, & \text { if } \widetilde{v} \neq \widehat{v} ;=0 \text {, otherwise, } \\
d(t)=\frac{-\widetilde{u}^{-q_{2}}(1, t)-\left(-\widehat{u}^{-q_{2}}(1, t)\right)}{\widetilde{u}(1, t)-\widehat{u}(1, t)}, & \text { if } \widetilde{u} \neq \widehat{u} ;=0 \text {, otherwise. }
\end{array}
$$

For any fixed $\tau \in(0, T)$, we will show that $\Psi \geq 0$ and $\Theta \geq 0$ for $0 \leq x \leq 1$ and $0 \leq t \leq \tau$. For contradiction, we assume that $\Theta$ has a negative minimum in $[0,1] \times[0, \tau]$ and $\min _{[0,1] \times[0, \tau]} \Theta \leq$ $\min _{[0,1] \times[0, \tau]} \Psi$. Let $\bar{\Theta}(x, t)=e^{-M t-L x^{2}} \Theta(x, t)$ and $\bar{\Psi}(x, t)=e^{-M t-L x^{2}} \Psi(x, t)$, where

$$
L=\max _{0 \leq t \leq \tau} c(t) / 2, M=2 L+4 L^{2}+\max _{[0,1] \times[0, \tau]} a(x, t)+\max _{[0,1] \times[0, \tau]} b(x, t) .
$$

Then $\bar{\Theta}$ and $\bar{\Psi}$ satisfies

$$
\begin{array}{ll}
\bar{\Theta}_{t} \geq \bar{\Theta}_{x x}+4 L x \bar{\Theta}_{x}+\left(2 L+4 L^{2} x^{2}-M\right) \bar{\Theta}+a(x, t) \bar{\Psi}, & 0<x<1,0<t<\tau, \\
\bar{\Psi}_{t} \geq \bar{\Psi}_{x x}+4 L x \bar{\Psi}_{x}+\left(2 L+4 L^{2} x^{2}-M\right) \bar{\Psi}+b(x, t) \bar{\Theta}, & 0<x<1,0<t<\tau .
\end{array}
$$

Since $\bar{\Theta} \geq-\delta$ and $\bar{\Psi} \geq-\delta$ on the boundary $([0,1] \times\{0\}) \cup(\{0,1\} \times(0, \tau])$, where $-\delta:=\min _{[0,1] \times[0, \tau]}$ $\bar{\Theta}<0$, it follows from the strong maximum principle for weakly coupled parabolic systems 
(cf. Theorem 15 of Chapter 3 in [7]) that $\Theta$ cannot assume its negative minimum in the interior. Hence $\bar{\Theta}>-\delta$ in $(0,1) \times(0, \tau]$. Let $\left(x_{0}, t_{0}\right)$ be a minimum point on the boundary $\{0,1\} \times(0, \tau]$. Since $\bar{\Theta}_{x}(0, t) \leq 0,0<t \leq \tau$, the same strong maximum principle implies that $x_{0}=1$ and $\bar{\Theta}_{x}\left(x_{0}, t_{0}\right)<0$. But,

$$
\bar{\Theta}_{x}\left(1, t_{0}\right)=-\left(c\left(t_{0}\right)-2 L\right) \Theta \geq 0,
$$

a contradiction. Then, we obtain that $\widetilde{u} \geq \widehat{u}$ and $\widetilde{v} \geq \widehat{v}$ in $[0,1] \times[0, T)$.

(b) It is clear from definition 2 that every solution of the problem (1.1) is an upper solution as well as a lower solution of the corresponding problem. If $\left(u^{*}, v^{*}\right)$ is a solution, then we get

$$
\begin{array}{lll}
\widetilde{u} \geq u^{*} & \text { and } & \widetilde{v} \geq v^{*}, \\
u^{*} \geq \widehat{u} & \text { and } & v^{*} \geq \widehat{v} .
\end{array}
$$

and

$$
\widetilde{u} \geq * \geq \widehat{u} \quad \text { and } \quad \widetilde{v} \geq v^{*} \geq \widehat{v},
$$

in $[0,1] \times[0, T)$ from Lemma $1-(a)$.

For a given pair of ordered upper and lower solutions $(\widetilde{u}, \widetilde{v})$ and $(\widehat{u}, \widehat{v})$ we set

$$
\begin{aligned}
S_{1} & =\{u \in C([0,1] \times[0, T)): \widehat{u} \leq u \leq \widetilde{u}\}, \\
S_{2} & =\{v \in C([0,1] \times[0, T)): \widehat{v} \leq v \leq \widetilde{v}\}, \\
S_{1} \times S_{2} & =\{(u, v) \in C([0,1] \times[0, T)) \times C([0,1] \times[0, T)):(\widehat{u}, \widehat{v}) \leq(u, v) \leq(\widetilde{u}, \widetilde{v})\} .
\end{aligned}
$$

Let

$$
\begin{aligned}
& f_{1}(x, t, v(x, t))=(1-v(x, t))^{-p_{1}}, g_{1}(x, t, v(x, t))=-v^{-q_{1}}(x, t), \\
& f_{2}(x, t, u(x, t))=(1-u(x, t))^{-p_{2}}, g_{2}(x, t, u(x, t))=-u^{-q_{2}}(x, t) .
\end{aligned}
$$

Throughout this section we make the following hypothesis on the above functions in the problem (1.1);

$\left(H_{1}\right)$-(i) The functions $f_{1}(x, t, \cdot), f_{2}(x, t, \cdot)$ are in $C^{\alpha, \alpha / 2}([0,1] \times[0, T))$ and $g_{1}(x, t, \cdot), g_{2}(x, t, \cdot)$ are in $C^{1+\alpha,(1+\alpha) / 2}(\{1\} \times(0, T))$, respectively.

$\left(H_{1}\right)$-(ii) Let $f_{1}(\cdot, v)$ and $g_{1}(\cdot, v)$ are $C^{1}$-functions of $v$ for $v \in S_{2}$, and $f_{2}(\cdot, u)$ and $g_{2}(\cdot, u)$ are $C^{1}$-functions of $u$ for $u \in S_{1}$, respectively. Also,

$$
\begin{cases}\left(f_{1}\right)_{v}(x, t, v) \geq 0 \text { for } v \in S_{2}, & (x, t) \in[0,1] \times[0, T), \\ \left(f_{2}\right)_{u}(x, t, u) \geq 0 \text { for } u \in S_{1}, & (x, t) \in[0,1] \times[0, T), \\ \left(g_{1}\right)_{v}(x, t, v) \geq 0 \text { for } v \in S_{2}, & (x, t) \in\{1\} \times(0, T), \\ \left(g_{2}\right)_{u}(x, t, u) \geq 0 \text { for } u \in S_{1}, & (x, t) \in\{1\} \times(0, T) .\end{cases}
$$


The condition (2.1) implies that $f_{1}(\cdot, v), g_{1}(\cdot, v)$ are non-decreasing in $v$ and $f_{2}(\cdot, u), g_{2}(\cdot, u)$ are non-decreasing in $u$, respectively, which is crucial for the construction of monotone sequences.

Next, we are going to construct monotone sequences of functions which give the estimation of the solution $(u, v)$ of the problem (1.1). Specifically, by starting from any initial iteration $\left(u^{0}, v^{0}\right)$ we can construct a sequence $\left\{u^{(k)}, v^{(k)}\right\}$ from the linear iteration process

$$
\begin{cases}u_{t}^{(k)}-u_{x x}^{(k)}=f_{1}\left(x, t, v^{(k-1)}\right), & 0<x<1,0<t<T, \\ v_{t}^{(k)}-v_{x x}^{(k)}=f_{2}\left(x, t, u^{(k-1)}\right), & 0<x<1,0<t<T, \\ u_{x}^{(k)}(0, t)=0, u_{x}^{(k)}(1, t)=g_{1}\left(1, t, v^{(k-1)}\right), & 0<t<T, \\ v_{x}^{(k)}(0, t)=0, v_{x}^{(k)}(1, t)=g_{2}\left(1, t, u^{(k-1)}\right), & 0<t<T, \\ u^{(k)}(x, 0)=u_{0}(x), v^{(k)}(x, 0)=v_{0}(x), & 0 \leq x \leq 1 .\end{cases}
$$

It is clear that the sequence governed by (2.2) is well defined and can be obtained by solving a linear initial boundary value problem. Starting from initial iteration $\left(u^{0}, v^{0}\right)=(\widetilde{u}, \widetilde{v})$ and $\left(u^{0}, v^{0}\right)=(\widehat{u}, \widehat{v})$, we define two sequences of the functions $\left\{\bar{u}^{(k)}, \bar{v}^{(k)}\right\}$ and $\left\{\underline{u}^{(k)}, \underline{v}^{(k)}\right\}$ for $k=$ $1,2, \ldots$ respectively, and refer to them as maximal and minimal sequences, respectively, where those functions satisfy the above linear problem.

Lemma 2. The sequences $\left\{\bar{u}^{(k)}, \bar{v}^{(k)}\right\},\left\{\underline{u}^{(k)}, \underline{v}^{(k)}\right\}$ possess the monotone property

$$
(\widehat{u}, \widehat{v}) \leq\left(\underline{u}^{(k)}, \underline{v}^{(k)}\right) \leq\left(\underline{u}^{(k+1)}, \underline{v}^{(k+1)}\right) \leq\left(\bar{u}^{(k+1)}, \bar{v}^{(k+1)}\right) \leq\left(\bar{u}^{(k)}, \bar{v}^{(k)}\right) \leq(\widetilde{u}, \widetilde{v})
$$

for $(x, t) \in[0,1] \times[0, T)$ and every $k=1,2, \ldots$

Proof. Let $\mu=\widetilde{u}-\bar{u}^{(1)}$ and $\lambda=\widetilde{v}-\bar{v}^{(1)}$. From (2.2) and Definition 1, we get

$$
\begin{aligned}
\mu_{t}-\mu_{x x} & =\widetilde{u}_{t}-\widetilde{u}_{x x}-f_{1}(x, t, \widetilde{v}) \geq 0, & & 0<x<1,0<t<T, \\
\lambda_{t}-\lambda_{x x} & =\widetilde{v}_{t}-\widetilde{v}_{x x}-f_{2}(x, t, \widetilde{u}) \geq 0, & & 0<x<1,0<t<T, \\
\mu_{x}(0, t) & =0, \mu_{x}(1, t)=\widetilde{u}_{x}(1, t)-g_{1}(1, t, \widetilde{v}) \geq 0, & & 0<t<T, \\
\lambda_{x}(0, t) & =0, \lambda_{x}(1, t)=\widetilde{v}_{x}(1, t)-g_{2}(1, t, \widetilde{u}) \geq 0, & & 0<t<T, \\
\mu(x, 0) & =\widetilde{u}(x, 0)-u_{0}(x) \geq 0, \lambda(x, 0)=\widetilde{v}(x, 0)-v_{0}(x) \geq 0, & & 0 \leq x \leq 1 .
\end{aligned}
$$

From Maximum principle and Hopf's Lemma for parabolic equations, we get $\mu, \lambda \geq 0$ for $(x, t) \in[0,1] \times[0, T)$, i.e. $\bar{u}^{(1)} \leq \widetilde{u}$ and $\bar{v}^{(1)} \leq \widetilde{v}$. Similarly, using the property of a lower solution, we obtain $\underline{u}^{(1)} \geq \widehat{u}$ and $\underline{v}^{(1)} \geq \widehat{v}$.

Let $\mu^{(1)}=\bar{u}^{(1)}-\underline{u}^{(1)}$ and $\lambda^{(1)}=\bar{v}^{(1)}-\underline{v}^{(1)}$. From (2.1) and (2.2), we get

$$
\mu_{t}^{(1)}-\mu_{x x}^{(1)}=f_{1}(x, t, \widetilde{v})-f_{1}(x, t, \widehat{v}) \geq 0, \quad 0<x<1,0<t<T,
$$




$$
\begin{aligned}
\lambda_{t}^{(1)}-\lambda_{x x}^{(1)} & =f_{2}(x, t, \widetilde{u})-f_{2}(x, t, \widehat{u}) \geq 0, & & 0<x<1,0<t<T, \\
\mu_{x}^{(1)}(0, t) & =0, \mu_{x}^{(1)}(1, t)=g_{1}(1, t, \widetilde{v})-g_{1}(1, t, \widehat{v}) \geq 0, & & 0<t<T, \\
\lambda_{x}^{(1)}(0, t) & =0, \lambda_{x}^{(1)}(1, t)=g_{2}(1, t, \widetilde{u})-g_{2}(1, t, \widehat{u}) \geq 0, & & 0<t<T, \\
\mu^{(1)}(x, 0) & =u_{0}(x)-u_{0}(x)=0, \lambda^{(1)}(x, 0)=v_{0}(x)-v_{0}(x)=0, & & 0 \leq x \leq 1 .
\end{aligned}
$$

From Maximum principle and Hopf's Lemma for parabolic equations, we get $\mu^{(1)}, \lambda^{(1)} \geq 0$ for $(x, t) \in[0,1] \times[0, T)$, i.e. $\underline{u}^{(1)} \leq \bar{u}^{(1)}$ and $\underline{v}^{(1)} \leq \bar{v}^{(1)}$. Therefore,

$$
(\widehat{u}, \widehat{v}) \leq\left(\underline{u}^{(1)}, \underline{v}^{(1)}\right) \leq\left(\bar{u}^{(1)}, \bar{v}^{(1)}\right) \leq(\widetilde{u}, \widetilde{v})
$$

for $(x, t) \in[0,1] \times[0, T)$.

Assume that

$$
\left(\underline{u}^{(k-1)}, \underline{v}^{(k-1)}\right) \leq\left(\underline{u}^{(k)}, \underline{v}^{(k)}\right) \leq\left(\bar{u}^{(k)}, \bar{v}^{(k)}\right) \leq\left(\bar{u}^{(k-1)}, \bar{v}^{(k-1)}\right)
$$

for $(x, t) \in[0,1] \times[0, T)$ and for some integer $k>1$. Let $\mu^{(k)}=\bar{u}^{(k)}-\bar{u}^{(k+1)}$ and $\lambda^{(k)}=\bar{v}^{(k)}-\bar{v}^{(k+1)}$. From (2.1) and (2.2), we get

$$
\begin{aligned}
\mu_{t}^{(k)}-\mu_{x x}^{(k)} & =f_{1}\left(x, t, \bar{v}^{(k-1)}\right)-f_{1}\left(x, t, \bar{v}^{(k)}\right) \geq 0, & & 0<x<1,0<t<T, \\
\lambda_{t}^{(k)}-\lambda_{x x}^{(k)} & =f_{2}\left(x, t, \bar{u}^{(k-1)}\right)-f_{2}\left(x, t, \bar{u}^{(k)}\right) \geq 0, & & 0<x<1,0<t<T, \\
\mu_{x}^{(k)}(0, t) & =0, \mu_{x}^{(k)}(1, t)=g_{1}\left(1, t, \bar{v}^{(k-1)}\right)-g_{1}\left(1, t, \bar{v}^{(k)}\right) \geq 0, & & 0<t<T, \\
\lambda_{x}^{(k)}(0, t) & =0, \lambda_{x}^{(k)}(1, t)=g_{2}\left(1, t, \bar{u}^{(k-1)}\right)-g_{2}\left(1, t, \bar{u}^{(k)}\right) \geq 0, & & 0<t<T, \\
\mu^{(k)}(x, 0) & =0, \lambda^{(k)}(x, 0)=0, & & 0 \leq x \leq 1 .
\end{aligned}
$$

From Maximum principle and Hopf's Lemma for parabolic equations, we get $\mu^{(k)}, \lambda^{(k)} \geq 0$ for $(x, t) \in[0,1] \times[0, T)$, i.e. $\bar{u}^{(k+1)} \leq \bar{u}^{(k)}$ and $\bar{v}^{(k+1)} \leq \bar{v}^{(k)}$. A similar argument gives $\underline{u}^{(k+1)} \geq$ $\underline{u}^{(k)}$ and $\underline{v}^{(k+1)} \geq \underline{v}^{(k)}$ and $\bar{u}^{(k+1)} \geq \underline{u}^{(k+1)}$ and $\bar{v}^{(k+1)} \geq \underline{v}^{(k+1)}$. Therefore, it follows from the mathematical induction, the lemma holds.

Lemma 3. For each positive integer $k,\left(\bar{u}^{(k)}, \bar{v}^{(k)}\right)$ is an upper solution, $\left(\underline{u}^{(k)}, \underline{v}^{(k)}\right)$ is a lower solution, $\underline{u}^{(k)} \leq \bar{u}^{(k)}$ and $\underline{v}^{(k)} \leq \bar{v}^{(k)}$ for $(x, t) \in[0,1] \times[0, T)$.

Proof. From (2.1), (2.2) and Lemma 2, $\left(\bar{u}^{(k)}, \bar{v}^{(k)}\right)$ satisfies

$$
\begin{aligned}
& \bar{u}_{t}^{(k)}-\bar{u}_{x x}^{(k)}=f_{1}\left(x, t, \bar{v}^{(k-1)}\right)=f_{1}\left(x, t, \bar{v}^{(k-1)}\right)-f_{1}\left(x, t, \bar{v}^{(k)}\right)+f_{1}\left(x, t, \bar{v}^{(k)}\right) \geq f_{1}\left(x, t, \bar{v}^{(k)}\right), \\
& \bar{v}_{t}^{(k)}-\bar{v}_{x x}^{(k)}=f_{2}\left(x, t, \bar{u}^{(k-1)}\right)=f_{2}\left(x, t, \bar{u}^{(k-1)}\right)-f_{2}\left(x, t, \bar{u}^{(k)}\right)+f_{2}\left(x, t, \bar{u}^{(k)}\right) \geq f_{2}\left(x, t, \bar{u}^{(k)}\right), \\
& \bar{u}_{x}^{(k)}(0, t)=0, \bar{v}_{x}^{(k)}(0, t)=0, \\
& \bar{u}_{x}^{(k)}(1, t)=g_{1}\left(1, t, \bar{v}^{(k-1)}\right)=g_{1}\left(1, t, \bar{v}^{(k-1)}\right)-g_{1}\left(1, t, \bar{v}^{(k)}\right)+g_{1}\left(1, t, \bar{v}^{(k)}\right) \geq g_{1}\left(1, t, \bar{v}^{(k)}\right)
\end{aligned}
$$




$$
\begin{aligned}
& \bar{v}_{x}^{(k)}(1, t)=g_{2}\left(1, t, \bar{u}^{(k-1)}\right)=g_{2}\left(1, t, \bar{u}^{(k-1)}\right)-g_{2}\left(1, t, \bar{u}^{(k)}\right)+g_{2}\left(1, t, \bar{u}^{(k)}\right) \geq g_{2}\left(1, t, \bar{u}^{(k)}\right) \\
& \bar{u}^{(k)}(x, 0)=u_{0}(x), \bar{v}^{(k)}(x, 0)=v_{0}(x), 0 \leq x \leq 1
\end{aligned}
$$

and $\left(\underline{u}^{(k)}, \underline{v}^{(k)}\right)$ satisfies

$$
\begin{aligned}
& \underline{u}_{t}^{(k)}-\underline{u}_{x x}^{(k)}=f_{1}\left(x, t, \underline{v}^{(k-1)}\right)=f_{1}\left(x, t, \underline{v}^{(k-1)}\right)-f_{1}\left(x, t, \underline{v}^{(k)}\right)+f_{1}\left(x, t, \underline{v}^{(k)}\right) \leq f_{1}\left(x, t, \underline{v}^{(k)}\right), \\
& \underline{v}_{t}^{(k)}-\underline{v}_{x x}^{(k)}=f_{2}\left(x, t, \underline{u}^{(k-1)}\right)=f_{2}\left(x, t, \underline{u}^{(k-1)}\right)-f_{2}\left(x, t, \underline{u}^{(k)}\right)+f_{2}\left(x, t, \underline{u}^{(k)}\right) \leq f_{2}\left(x, t, \underline{u}^{(k)}\right), \\
& \underline{u}_{x}^{(k)}(0, t)=0, \underline{v}_{x}^{(k)}(0, t)=0, \\
& \underline{u}_{x}^{(k)}(1, t)=g_{1}\left(1, t, \underline{v}^{(k-1)}\right)=g_{1}\left(1, t, \underline{v}^{(k-1)}\right)-g_{1}\left(1, t, \underline{v}^{(k)}\right)+g_{1}\left(1, t, \underline{v}^{(k)}\right) \leq g_{1}\left(1, t, \underline{v}^{(k)}\right) \\
& \underline{v}_{x}^{(k)}(1, t)=g_{2}\left(1, t, \underline{u}^{(k-1)}\right)=g_{2}\left(1, t, \underline{u}^{(k-1)}\right)-g_{2}\left(1, t, \underline{u}^{(k)}\right)+g_{2}\left(1, t, \underline{u}^{(k)}\right) \leq g_{2}\left(1, t, \underline{u}^{(k)}\right) \\
& \underline{u}^{(k)}(x, 0)=u_{0}(x), \underline{v}^{(k)}(x, 0)=v_{0}(x), 0 \leq x \leq 1 .
\end{aligned}
$$

From Lemma 2 and above inequalities, the functions $\left(\bar{u}^{(k)}, \bar{v}^{(k)}\right)$ and $\left(\underline{u}^{(k)}, \underline{v}^{(k)}\right)$ are ordered upper and lower solutions of the problem (2.2).

We have the following existence theorem for the problem (1.1) via Lemma 2 and Lemma 3.

Theorem 1. Let $(\widetilde{u}, \widetilde{v}),(\widehat{u}, \widehat{v})$ be a pair of ordered upper and lower solutions of the problem (1.1), and let Hypothesis $\left(H_{1}\right)$ hold. Then the sequences $\left\{\bar{u}^{(k)}, \bar{v}^{(k)}\right\},\left\{\underline{u}^{(k)}, \underline{v}^{(k)}\right\}$ given by the problem (2.2) with $\left(u^{0}, v^{0}\right)=(\widetilde{u}, \widetilde{v})$ and $\left(u^{0}, v^{0}\right)=(\widehat{u}, \widehat{v})$ convergence monotonically to a maximal solution $(\bar{u}, \bar{v})$ and minimal solution $(\underline{u}, \underline{v})$ of the problem (1.1), respectively. Further,

$$
(\widehat{u}, \widehat{v}) \leq\left(\underline{u}^{(k)}, \underline{v}^{(k)}\right) \leq\left(\underline{u}^{(k+1)}, \underline{v}^{(k+1)}\right) \leq(\underline{u}, \underline{v}) \leq(\bar{u}, \bar{v}) \leq\left(\bar{u}^{(k+1)}, \bar{v}^{(k+1)}\right) \leq\left(\bar{u}^{(k)}, \bar{v}^{(k)}\right) \leq(\widetilde{u}, \widetilde{v})
$$

for $(x, t) \in[0,1] \times[0, T)$ and each positive integer $k$. Furthermore $(\underline{u}, \underline{v})=(\bar{u}, \bar{v})\left(\equiv\left(u^{*}, v^{*}\right)\right)$ then $\left(u^{*}, v^{*}\right)$ is the unique solution of the problem (1.1) in $S_{1} \times S_{2}$.

Proof. The pointwise limits

$$
\lim _{k \rightarrow \infty}\left(\bar{u}^{(k)}(x, t), \bar{v}^{(k)}(x, t)\right)=(\bar{u}(x, t), \bar{v}(x, t)), \lim _{k \rightarrow \infty}\left(\underline{u}^{(k)}(x, t), \underline{v}^{(k)}(x, t)\right)=(\underline{u}(x, t), \underline{v}(x, t))
$$

exist and satisfy relation (2.3). Indeed, the sequence $\left\{\bar{u}^{(k)}, \bar{v}^{(k)}\right\}$ is monotone nonincreasing which is bounded from below, while the sequence $\left\{\underline{u}^{(k)}, \underline{v}^{(k)}\right\}$ is monotone nondecreasing and is bounded from Lemma 2.

Let $\Theta=\underline{u}(x, t)-\bar{u}(x, t)$ and $\Psi=\underline{v}(x, t)-\bar{v}(x, t)$. From (2.3), we have $\underline{u}(x, t) \leq \bar{u}(x, t)$ and $\underline{v}(x, t) \leq \bar{v}(x, t)$ for $(x, t) \in[0,1] \times[0, T)$. Also, then $\Theta(x, t)$ and $\Psi(x, t)$ satisfies

$$
\begin{array}{rlrl}
\Theta_{t}-\Theta_{x x} & =f_{1}(x, t, \underline{v})-f_{1}(x, t, \bar{v}), & 0<x<1,0<t<T, \\
\Psi_{t}-\Psi_{x x}=f_{2}(x, t, \underline{u})-f_{2}(x, t, \bar{u}), & 0<x<1,0<t<T,
\end{array}
$$




$$
\begin{aligned}
\Theta_{x}(0, t) & =0, \Psi_{x}(0, t)=0, & & 0<t<T, \\
\Theta_{x}(1, t) & =g_{1}(1, t, \underline{v})-g_{1}(1, t, \bar{v}), & & 0<t<T, \\
\Psi_{x}(1, t) & =g_{2}(1, t, \underline{u})-g_{2}(1, t, \bar{u}), & & 0<t<T, \\
\Theta(x, 0) & =0, \Psi(x, 0)=0, & & 0 \leq x \leq 1 .
\end{aligned}
$$

By using Lemma 1-(a) and Lemma 4-(a) process, $\Theta \geq 0$ and $\Psi \geq 0$ for $(x, t) \in[0,1] \times[0, T)$, i.e. $\underline{u}(x, t) \geq \bar{u}(x, t)$ and $\underline{v}(x, t) \geq \bar{v}(x, t)$. Then, we get $\underline{u}(x, t)=\bar{u}(x, t)$ and $\underline{v}(x, t)=\bar{v}(x, t)$.

If $\left(u^{*}, v^{*}\right)$ is any other solution in $S_{1} \times S_{2}$, then we get from Lemma 3,

$$
\begin{aligned}
& \bar{u} \geq u^{*} \quad \text { and } \quad \bar{v} \geq v^{*} \text {, } \\
& u^{*} \geq \underline{u} \text { and } v^{*} \geq \underline{v} \text {, }
\end{aligned}
$$

and

$$
\bar{u} \geq u^{*} \geq \underline{u} \quad \text { and } \quad \bar{v} \geq v^{*} \geq \underline{v}
$$

in $[0,1] \times[0, T)$. This implies that

$$
\bar{u}=u^{*}=\underline{u} \quad \text { and } \quad \bar{v}=v^{*}=\underline{v}
$$

and hence $\left(u^{*}, v^{*}\right)$ is the unique solution of the problem (1.1).

\section{The First Problem}

In this section, we investigate quenching behavior of solution of semilinear reaction-diffusion system (1.1) with (1.2)-(1.3) initial conditions.

Remark 1. If $\left(u_{0}, v_{0}\right)$ satisfies (1.3), then we get $u_{x}<0$ and $v_{x}<0$ in $(0,1] \times(0, T)$ by the maximum principle. Thus we get $u(0, t)=\max _{0 \leq x \leq 1} u(x, t)$ and $v(0, t)=\max _{0 \leq x \leq 1} v(x, t)$.

Lemma 4. If $\left(u_{0}, v_{0}\right)$ satisfies (1.2), then $u_{t}(x, t) \geq 0$ and $v_{t}(x, t) \geq 0$ in $[0,1] \times[0, T)$.

Proof. We give the proof by utilizing Lemma 2.1 in [2]. Let $\Theta=u_{t}(x, t)$ and $\Psi=v_{t}(x, t)$. Then $\Theta(x, t)$ satisfies

$$
\begin{aligned}
& \Theta_{t}=\Theta_{x x}+p_{1}(1-v)^{-p_{1}-1} \Psi, 0<x<1,0<t<T, \\
& \Theta_{x}(0, t)=0, \Theta_{x}(1, t)=q_{1} v^{-q_{1}-1}(1, t) \Psi(1, t), 0<t<T, \\
& \Theta(x, 0)=u_{x x}(x, 0)+(1-v(x, 0))^{-p_{1}} \geq 0,0 \leq x \leq 1,
\end{aligned}
$$

and $\Psi(x, t)$ satisfies

$$
\begin{aligned}
& \Psi_{t}=\Psi_{x x}+p_{2}(1-u)^{-p_{2}-1} \Theta, 0<x<1,0<t<T, \\
& \Psi_{x}(0, t)=0, \Psi_{x}(1, t)=q_{2} u^{-q_{2}-1}(1, t) \Theta(1, t), 0<t<T, \\
& \Psi(x, 0)=v_{x x}(x, 0)+(1-u(x, 0))^{-p_{2}} \geq 0,0 \leq x \leq 1 .
\end{aligned}
$$


For any fixed $\tau \in(0, T)$, we will show that $\Psi \geq 0$ and $\Theta \geq 0$ for $0 \leq x \leq 1$ and $0 \leq t \leq \tau$. For contradiction, we assume that $\Theta$ has a negative minimum in $[0,1] \times[0, \tau]$ and $\min _{[0,1] \times[0, \tau]} \Theta \leq$ $\min _{[0,1] \times[0, \tau]} \Psi$. Let $\bar{\Theta}(x, t)=e^{-M t-L x^{2}} \Theta(x, t)$ and $\bar{\Psi}(x, t)=e^{-M t-L x^{2}} \Psi(x, t)$, where

$$
\begin{aligned}
L & =\max _{0 \leq t \leq \tau}\left(\frac{1}{2} q_{1} v^{-q_{1}-1}(1, t)\right), \\
M & =2 L+4 L^{2}+\max _{[0,1] \times[0, \tau]}\left(p_{1}(1-v(x, t))^{-p_{1}-1}\right)+\max _{[0,1] \times[0, \tau]}\left(p_{2}(1-u(x, t))^{-p_{2}-1}\right) .
\end{aligned}
$$

Then $\bar{\Theta}$ and $\bar{\Psi}$ satisfies

$$
\begin{aligned}
& \bar{\Theta}_{t}=\bar{\Theta}_{x x}+4 L x \bar{\Theta}_{x}+\left(2 L+4 L^{2} x^{2}-M\right) \bar{\Theta}+p_{1}(1-v(x, t))^{-p_{1}-1} \bar{\Psi}, \quad 0<x<1,0<t<\tau, \\
& \bar{\Psi}_{t}=\bar{\Psi}_{x x}+4 L x \bar{\Psi}_{x}+\left(2 L+4 L^{2} x^{2}-M\right) \bar{\Psi}+p_{2}(1-u(x, t))^{-p_{2}-1} \bar{\Theta}, \quad 0<x<1,0<t<\tau .
\end{aligned}
$$

Since $\bar{\Theta} \geq-\delta$ and $\bar{\Psi} \geq-\delta$ on the boundary $([0,1] \times\{0\}) \cup(\{0,1\} \times(0, \tau])$, where $-\delta:=\min _{[0,1] \times[0, \tau]}$ $\bar{\Theta}<0$, it follows from the strong maximum principle for weakly coupled parabolic systems (cf. Theorem 15 of Chapter 3 in [7]) that $\bar{\Theta}$ cannot assume its negative minimum in the interior. Hence $\bar{\Theta}>-\delta$ in $(0,1) \times(0, \tau]$. Let $\left(x_{0}, t_{0}\right)$ be a minimum point on the boundary $\{0,1\} \times(0, \tau]$. Since $\bar{\Theta}_{x}(0, t) \leq 0,0<t \leq \tau$, the same strong maximum principle implies that $x_{0}=1$ and $\bar{\Theta}_{x}\left(x_{0}, t_{0}\right)<0$. But,

$$
\bar{\Theta}_{x}\left(1, t_{0}\right)=\left(q_{1} v^{-q_{1}-1}\left(1, t_{0}\right)-2 L\right) \Theta=-\left(q_{1} v^{-q_{1}-1}\left(1, t_{0}\right)-2 L\right) \delta \geq 0,
$$

a contradiction. Then, we obtain that $\bar{\Theta} \geq 0$ and $\bar{\Psi} \geq 0$ in $[0,1] \times[0, \tau]$. Thus, $u_{t}(x, t) \geq 0$ and $v_{t}(x, t) \geq 0$ in $[0,1] \times[0, T)$.

Theorem 2. If $\left(u_{0}, v_{0}\right)$ satisfies (1.2), then there exists a finite time $T$, such that the solution $(u, v)$ of the problem (1.1) quenches at time $T$.

Proof. Assume that $\left(u_{0}, v_{0}\right)$ satisfies (1.2). Then there exist

$$
\begin{aligned}
& w_{1}=-v^{-q_{1}}(1,0)+\int_{0}^{1}(1-v(x, 0))^{-p_{1}} d x>0, \\
& w_{2}=-u^{-q_{2}}(1,0)+\int_{0}^{1}(1-u(x, 0))^{-p_{2}} d x>0 .
\end{aligned}
$$

Define; $m_{1}(t)=\int_{0}^{1}(1-u(x, t)) d x$ and $m_{2}(t)=\int_{0}^{1}(1-v(x, t)) d x, 0<t<T$. Then, we get

$$
\begin{aligned}
& m_{1}^{\prime}(t)=v^{-q_{1}}(1, t)-\int_{0}^{1}(1-v(x, t))^{-p_{1}} d x \leq-w_{1}, \\
& m_{2}^{\prime}(t)=u^{-q_{2}}(1, t)-\int_{0}^{1}(1-u(x, t))^{-p_{2}} d x \leq-w_{2},
\end{aligned}
$$

by Lemma 4 . Thus, $m_{1}(t) \leq m_{1}(0)-w_{1} t$ and $m_{2}(t) \leq m_{2}(0)-w_{2} t$; which means that $m_{1}\left(T_{0}\right)=$ 0 or $m_{2}\left(T_{0}\right)=0$ for some $T_{0}=\min \left(\frac{m_{1}(0)}{w_{1}}, \frac{m_{2}(0)}{w_{2}}\right),\left(0<T \leq T_{0}\right)$. Then, $(u, v)$ quenches in finite time. 
Theorem 3. If $\left(u_{0}, v_{0}\right)$ satisfies (1.3), then $x=0$ is the only quenching point.

Proof. Define

$$
J(x, t)=u_{x}+\varepsilon\left(b_{2}-x\right) \text { in }\left[b_{1}, b_{2}\right] \times[\tau, T),
$$

where $b_{2} \in(0,1], b_{1} \in\left(0, b_{2}\right), \tau \in(0, T)$ and $\varepsilon$ is a positive constant to be specified later. Then, $J(x, t)$ satisfies

$$
J_{t}-J_{x x}=p_{1}(1-v)^{-p_{1}-1} v_{x}<0 \text { in }\left(b_{1}, b_{2}\right) \times[\tau, T),
$$

since $v_{x}(x, t)<0$ in $(0,1] \times(0, T)$. Thus, $J(x, t)$ cannot attain a positive interior maximum by the maximum principle. Further, if $\varepsilon$ is small enough, $J(x, \tau)<0$ since $u_{x}(x, t)<0$ in $(0,1] \times(0, T)$. Furthermore, if $\varepsilon$ is small enough,

$$
\begin{aligned}
& J\left(b_{1}, t\right)=u_{x}\left(b_{1}, t\right)+\varepsilon\left(b_{2}-b_{1}\right)<0, \\
& J\left(b_{2}, t\right)=u_{x}\left(b_{2}, t\right)<0,
\end{aligned}
$$

for $t \in(\tau, T)$. By the maximum principle, we obtain that $J(x, t)<0$, i.e. $u_{x}<-\varepsilon\left(b_{2}-x\right)$ for $(x, t) \in\left[b_{1}, b_{2}\right] \times[\tau, T)$. Integrating this with respect to $x$ from $b_{1}$ to $b_{2}$, we have

$$
u\left(b_{2}, t\right)<u\left(b_{1}, t\right)-\frac{\varepsilon\left(b_{2}-b_{1}\right)^{2}}{2}<1-\frac{\varepsilon\left(b_{2}-b_{1}\right)^{2}}{2}<1 .
$$

So $u$ does not quench in $(0,1]$. Similarly, we show that $v$ does not quench in $(0,1]$. The theorem is proved.

Theorem 4. If $\left(u_{0}, v_{0}\right)$ satisfies (1.2)-(1.3), $\left(u_{t}, v_{t}\right)$ blows up at the quenching time.

Proof. Define

$$
\begin{aligned}
& J_{1}(x, t)=u_{t}-\varepsilon\left(x-b_{1}\right)(1-v)^{-p_{1}} \text { in }\left[b_{1}, b_{2}\right] \times[\tau, T), \\
& J_{2}(x, t)=v_{t}-\varepsilon\left(x-b_{1}\right)(1-u)^{-p_{2}} \text { in }\left[b_{1}, b_{2}\right] \times[\tau, T),
\end{aligned}
$$

where $b_{1} \in[0,1), b_{2} \in\left(b_{1}, 1\right], \tau \in(0, T)$ and $\varepsilon$ is a positive constant to be specified later. Then, $J_{1}(x, t)$ satisfies

$$
\begin{aligned}
& \left(J_{1}\right)_{t}-\left(J_{1}\right)_{x x}-p_{1}(1-v)^{-p_{1}-1} J_{2} \\
& =2 \varepsilon p_{1}\left(x-b_{1}\right)(1-v)^{-p_{1}-1} v_{x}+\varepsilon p_{1}\left(p_{1}+1\right)\left(x-b_{1}\right)(1-v)^{-p_{1}-2} v_{x}^{2}>0
\end{aligned}
$$

and $J_{2}(x, t)$ satisfies

$$
\begin{aligned}
& \left(J_{2}\right)_{t}-\left(J_{2}\right)_{x x}-p_{2}(1-u)^{-p_{2}-1} J_{1} \\
& =2 \varepsilon p_{2}\left(x-b_{1}\right)(1-u)^{-p_{2}-1} u_{x}+\varepsilon p_{2}\left(p_{2}+1\right)\left(x-b_{1}\right)(1-u)^{-p_{2}-2} u_{x}^{2}>0
\end{aligned}
$$


since $u_{x}(x, t), v_{x}(x, t)>0$ in $(0,1) \times(0, T) . J_{1}(x, \tau), J_{2}(x, \tau) \geq 0$ if $\varepsilon$ is small enough and Remark 2. Further, if $\varepsilon$ is small enough,

$$
\begin{aligned}
& J_{1}\left(b_{1}, t\right)=u_{t}\left(b_{1}, t\right)>0, J_{1}\left(b_{2}, t\right)=u_{t}\left(b_{2}, t\right)-\varepsilon\left(b_{2}-b_{1}\right)(1-v)^{-p_{1}}>0, \\
& J_{2}\left(b_{1}, t\right)=v_{t}\left(b_{1}, t\right)>0, J_{2}\left(b_{2}, t\right)=v_{t}\left(b_{2}, t\right)-\varepsilon\left(b_{2}-b_{1}\right)(1-u)^{-p_{2}}>0,
\end{aligned}
$$

for $t \in(\tau, T)$. By the maximum principle, we obtain that $J_{1}(x, t), J_{2}(x, t) \geq 0$ for $(x, t) \in[0,1] \times$ $[0, T)$. Namely, $u_{t} \geq \varepsilon\left(x-b_{1}\right)(1-v)^{-p_{1}}$ and $v_{t} \geq \varepsilon\left(x-b_{1}\right)(1-u)^{-p_{2}}$ for $(x, t) \in\left[b_{1}, b_{2}\right] \times[\tau, T)$, i.e. $u_{t} \geq \varepsilon x v(1-v)^{-p_{1}}$ and $v_{t} \geq \varepsilon x(1-u)^{-p_{2}}$ for $(x, t) \in[0,1] \times[\tau, T)$. For $x=1$, we get

$$
u_{t}(1, t) \geq \varepsilon(1-v(1, t))^{-p_{1}},
$$

and

$$
v_{t}(1, t) \geq \varepsilon(1-u(1, t))^{-p_{2}} .
$$

Thus, we get

$$
\begin{aligned}
& \lim _{t \rightarrow T^{-}} u_{t}(1, t) \geq \lim _{t \rightarrow T^{-}} \varepsilon(1-v(1, t))^{-p_{1}}=\infty, \\
& \lim _{t \rightarrow T^{-}} v_{t}(1, t) \geq \lim _{t \rightarrow T^{-}} \varepsilon(1-u(1, t))^{-p_{2}}=-\infty .
\end{aligned}
$$

The theorem is proved.

\section{The second problem}

In this section, we investigate quenching behavior of solution of semilinear reaction-diffusion system (1.1) with (1.3)-(1.4) initial conditions.

Remark 2. $\mathrm{f}\left(u_{0}, v_{0}\right)$ satisfies (1.4), then we get $u_{t}(x, t) \leq 0$ and $v_{t}(x, t) \leq 0$ in $[0,1] \times[0, T)$ (We can give the proof as proof of Lemma 4). Also, for any $(\xi, \eta) \in(0,1) \times(0, T)$, there exists a subset $\left[x_{1}, x_{2}\right] \times\left[t_{1}, t_{2}\right]$ of $(0,1) \times(0, T)$ such that $(\xi, \eta) \in\left[x_{1}, x_{2}\right] \times\left[t_{1}, t_{2}\right]$. Define, $H=u_{t}, K=v_{t}$ in $\left[x_{1}, x_{2}\right] \times\left[t_{1}, t_{2}\right]$. We get that

$$
\begin{aligned}
H_{t}-H_{x x} & =p_{1}(1-v)^{-p_{1}-1} K \text { in }\left(x_{1}, x_{2}\right) \times\left(t_{1}, t_{2}\right), \\
K_{t}-K_{x x} & =p_{2}(1-u)^{-p_{2}-1} H \text { in }\left(x_{1}, x_{2}\right) \times\left(t_{1}, t_{2}\right), \\
H, K & \leq 0 \text { on }\left[x_{1}, x_{2}\right] \times\left[t_{1}, t_{2}\right] .
\end{aligned}
$$

The strong maximum principle implies that either $H, K<0$ or $H, K \equiv 0$ in $\left(x_{1}, x_{2}\right) \times\left(t_{1}, t_{2}\right)$. Since $H, K \equiv 0$ contradicts to the fact that $u(x, t)$ and $v(x, t)$ is strictly decreasing $t$. Therefore, $u_{t}, v_{t}<0$. Because $(\xi, \eta)$ is arbitrary in $(0,1) \times(0, T)$, we have $u_{t}, v_{t}<0$ in $(0,1) \times(0, T)$.

Theorem 5. If $\left(u_{0}, v_{0}\right)$ satisfies (1.4), then there exists a finite time $T$, such that the solution $(u, v)$ of the problem (1.1) quenches at time $T$. 
Proof. Assume that $\left(u_{0}, v_{0}\right)$ satisfies (1.4). Then there exist

$$
\begin{aligned}
& w_{1}=v^{-q_{1}}(1,0)-\int_{0}^{1}(1-v(x, 0))^{-p_{1}} d x>0, \\
& w_{2}=u^{-q_{2}}(1,0)-\int_{0}^{1}(1-u(x, 0))^{-p_{2}} d x>0 .
\end{aligned}
$$

Define; $m_{1}(t)=\int_{0}^{1} u(x, t) d x$ and $m_{2}(t)=\int_{0}^{1} v(x, t) d x, 0<t<T$. Then, we get

$$
\begin{aligned}
& m_{1}^{\prime}(t)=-v^{-q_{1}}(1, t)+\int_{0}^{1}(1-v(x, t))^{-p_{1}} d x \leq-w_{1}, \\
& m_{2}^{\prime}(t)=-u^{-q_{2}}(1, t)+\int_{0}^{1}(1-u(x, t))^{-p_{2}} d x \leq-w_{2},
\end{aligned}
$$

by Remark 2 . Thus, $m_{1}(t) \leq m_{1}(0)-w_{1} t$ and $m_{2}(t) \leq m_{2}(0)-w_{2} t$; which means that $m_{1}\left(T_{0}\right)=$ 0 or $m_{2}\left(T_{0}\right)=0$ for some $T_{0}=\min \left(\frac{m_{1}(0)}{w_{1}}, \frac{m_{2}(0)}{w_{2}}\right),\left(0<T \leq T_{0}\right)$. Then, $(u, v)$ quenches in finite time.

Theorem 6. If $\left(u_{0}, v_{0}\right)$ satisfies (1.3), then $x=1$ is the only quenching point.

Proof. Define

$$
J(x, t)=u_{x}+\varepsilon\left(x-b_{1}\right) \text { in }\left[b_{1}, b_{2}\right] \times[\tau, T),
$$

where $b_{1} \in[0,1), b_{2} \in\left(b_{1}, 1\right], \tau \in(0, T)$ and $\varepsilon$ is a positive constant to be specified later. Then, $J(x, t)$ satisfies

$$
J_{t}-J_{x x}=p_{1}(1-v)^{-p_{1}-1} v_{x}<0 \text { in }\left(b_{1}, b_{2}\right) \times[\tau, T),
$$

since $v_{x}(x, t)<0$ in $(0,1] \times(0, T)$. Thus, $J(x, t)$ cannot attain a positive interior maximum by the maximum principle. Further, if $\varepsilon$ is small enough, $J(x, \tau)<0$ since $u_{x}(x, t)<0$ in $(0,1] \times(0, T)$. Furthermore, if $\varepsilon$ is small enough,

$$
\begin{aligned}
& J\left(b_{1}, t\right)=u_{x}\left(b_{1}, t\right)<0, \\
& J\left(b_{2}, t\right)=u_{x}\left(b_{2}, t\right)+\varepsilon\left(b_{2}-b_{1}\right)<0,
\end{aligned}
$$

for $t \in(\tau, T)$. By the maximum principle, we obtain that $J(x, t)<0$, i.e. $u_{x}<-\varepsilon\left(x-b_{1}\right)$ for $(x, t) \in\left[b_{1}, b_{2}\right] \times[\tau, T)$. Integrating this with respect to $x$ from $b_{1}$ to $b_{2}$, we have

$$
u\left(b_{2}, t\right)<u\left(b_{1}, t\right)-\frac{\varepsilon\left(b_{2}-b_{1}\right)^{2}}{2}
$$

and

$$
u\left(b_{1}, t\right)>u\left(b_{2}, t\right)+\frac{\varepsilon\left(b_{2}-b_{1}\right)^{2}}{2}>0 .
$$

So $u$ does not quench in $[0,1)$. Similarly, we get $v$ does not quench in $[0,1)$. 
Theorem 7. If $\left(u_{0}, v_{0}\right)$ satisfies (1.3)-(1.4), $\left(u_{t}, v_{t}\right)$ blows up at the quenching time.

Proof. Define

$$
\begin{aligned}
& J_{1}(x, t)=u_{t}+\varepsilon\left(x-b_{1}\right) v^{-q_{1}} \text { in }\left[b_{1}, b_{2}\right] \times[\tau, T), \\
& J_{2}(x, t)=v_{t}+\varepsilon\left(x-b_{1}\right) u^{-q_{2}} \text { in }\left[b_{1}, b_{2}\right] \times[\tau, T),
\end{aligned}
$$

where $b_{1} \in[0,1), b_{2} \in\left(b_{1}, 1\right], \tau \in(0, T)$ and $\varepsilon$ is a positive constant to be specified later. Then, $J_{1}(x, t)$ satisfies

$$
\begin{aligned}
& \left(J_{1}\right)_{t}-\left(J_{1}\right)_{x x}-p_{1}(1-v)^{-p_{1}-1} J_{2} \\
& =-\varepsilon q_{1}\left(x-b_{1}\right)(1-u)^{-p_{2}} v^{-q_{1}-1}+2 \varepsilon q_{1}\left(x-b_{1}\right) v^{-q_{1}-1} v_{x} \\
& \quad-\varepsilon q_{1}\left(q_{1}+1\right)\left(x-b_{1}\right) v^{-q_{1}-2} v_{x}^{2}-\varepsilon p_{1}\left(x-b_{1}\right)(1-v)^{-p_{1}-1} v^{-q_{1}}<0
\end{aligned}
$$

and $J_{2}(x, t)$ satisfies

$$
\begin{aligned}
& \left(J_{2}\right)_{t}-\left(J_{2}\right)_{x x}-p_{2}(1-u)^{-p_{2}-1} J_{1} \\
& =-\varepsilon q_{2}\left(x-b_{1}\right)(1-v)^{-p_{1}} u^{-q_{2}-1}+2 \varepsilon q_{2}\left(x-b_{1}\right) u^{-q_{2}-1} u_{x} \\
& \quad-\varepsilon q_{2}\left(q_{2}+1\right)\left(x-b_{1}\right) u^{-q_{2}-2} u_{x}^{2}-\varepsilon p_{2}\left(x-b_{1}\right)(1-u)^{-p_{2}-1} u^{-q_{2}}<0
\end{aligned}
$$

since $u_{x}(x, t), v_{x}(x, t)<0$ in $(0,1) \times(0, T) . J_{1}(x, \tau), J_{2}(x, \tau) \leq 0$ if $\varepsilon$ is small enough and Remark 2. Further, if $\varepsilon$ is small enough,

$$
\begin{aligned}
& J_{1}\left(b_{1}, t\right)=u_{t}\left(b_{1}, t\right)<0, J_{1}\left(b_{2}, t\right)=u_{t}\left(b_{2}, t\right)+\varepsilon\left(b_{2}-b_{1}\right) v^{-q_{1}}<0, \\
& J_{2}\left(b_{1}, t\right)=v_{t}\left(b_{1}, t\right)<0, J_{2}\left(b_{2}, t\right)=v_{t}\left(b_{2}, t\right)+\varepsilon\left(b_{2}-b_{1}\right) u^{-q_{2}}<0,
\end{aligned}
$$

for $t \in(\tau, T)$. By the maximum principle, we obtain that $J_{1}(x, t), J_{2}(x, t) \leq 0$ for $(x, t) \in[0,1] \times$ $[0, T)$. Namely, $u_{t} \leq-\varepsilon\left(x-b_{1}\right) v^{-q_{1}}(x, t)$ and $v_{t} \leq-\varepsilon\left(x-b_{1}\right) u^{-q_{2}}(x, t)$ for $(x, t) \in\left[b_{1}, b_{2}\right] \times$ $[\tau, T)$, i.e. $u_{t} \leq-\varepsilon x v^{-q_{1}}(x, t)$ and $v_{t} \leq-\varepsilon x u^{-q_{2}}(x, t)$ for $(x, t) \in[0,1] \times[\tau, T)$. For $x=1$, we get

$$
u_{t}(1, t) \leq-\varepsilon v^{-q_{1}}(1, t),
$$

and

$$
v_{t}(1, t) \leq-\varepsilon u^{-q_{2}}(1, t)
$$

Thus, we get

$$
\begin{gathered}
\lim _{t \rightarrow T^{-}} u_{t}(1, t) \leq \lim _{t \rightarrow T^{-}}-\varepsilon v^{-q_{1}}(1, t)=-\infty, \\
\lim _{t \rightarrow T^{-}} v_{t}(1, t) \leq \lim _{t \rightarrow T^{-}}-\varepsilon u^{-q_{2}}(1, t)=-\infty .
\end{gathered}
$$

The theorem is proved. 


\section{References}

[1] C. W. Chang, Y. H. Hsu and H. T. Liu, Quenching behavior of parabolic problems with localized reaction term, Mathematics and Statistics, 2 (2014), 48-53.

[2] S.-C. Fu and J.-S. Guo, Blow up for a semilinear reaction-diffusion system coupled in both equations and boundary conditions, J. Math. Anal. Appl., 276 (2002), 458-475.

[3] C. Mu, S. Zhou and D. Liu, Quenching for a reaction diffusion system with logarithmic singularity, Nonlinear Analysis, 71 (2009), 5599-5605.

[4] N. Ozalp and B. Selcuk, The quenching behavior of a nonlinear parabolic equation with a singular parabolic with a singular boundary condition, Hacettepe Journal of Mathematics and Statistics, doi: 10.15672/HJMS.2015449429.

[5] C. V. Pao, Quasilinear parabolic and elliptic equations with nonlinear boundary conditions, Nonlinear Analysis, 66 (2007), 639-662.

[6] C. V. Pao and W. H. Ruan, Positive solutions of quasilinear parabolic systems with nonlinear boundary conditions, J. Math. Anal. Appl., 333 (2007), 472-499.

[7] M. H. Protter and H. F. Weinberger, Maximum Principles in Differential Equations, Springer, New York, 1984.

[8] B. Selcuk and N. Ozalp, The quenching behavior of a semilinear heat equation with a singular boundary outflux, Quarterly of Applied Mathematics, 72 (2014), 747-752.

[9] S. Zheng and W. Wang, Non-simultaneous versus simultaneous quenching in a coupled nonlinear parabolic system, Nonlinear Analysis, 69 (2008), 2274-2285.

[10] J. Zhou, Y. He and C. Mu, Incomplete quenching of heat equations with absorption, Applicable Analysis, 87 (2008), 523-529.

Department of Computer Engineering, Karabuk University, Balıklarkayası Mevkii, 78050, Turkey.

E-mail: bselcuk@karabuk.edu.tr 